\title{
The DSC approach to study non-freezing water contents of hydrated hydroxypropylcellulose (HPC)
}

\section{A study over effects of viscosity and drug addition}

\author{
Przemysław Talik ${ }^{1}$ (i) $\cdot$ Urszula Hubicka $^{1}$ \\ Received: 28 July 2017 / Accepted: 3 December 2017 / Published online: 11 December 2017 \\ (C) The Author(s) 2017. This article is an open access publication
}

\begin{abstract}
The research consisted of investigation of non-freezable bound water (NFW) contents of raw hydroxypropylcellulose (HPC) Klucel ${ }^{\circledR}$ Pharm HF, MF, and LF by differential scanning calorimetry. Polymers (HF, MF and LF) used in the research differed significantly in their molecular mass and viscosity. Mixtures of HPC and low soluble salicylic acid or highly soluble sodium salicylate were used to examine the effect of counter-ions presence and drug solubility on NFW contents. The dependencies of determined enthalpies of melting $(\Delta H)$ and respective contents of water $W_{\mathrm{c}}$ (water fraction in the dry mass of HPC or HPC mixture) $\Delta H=\mathrm{f}\left(W_{\mathrm{c}}\right)$ were linear. The sought values of non-freezing water were calculated (by extrapolation) as the concentration at which $\Delta H=0$. It was found that the viscosity and molecular mass of the raw HPCs did not affect the contents of non-freezing water, which ranged between 0.54 and $0.51 \mathrm{~g} \mathrm{~g}^{-1}$ for all types LF, MF, HF. Poorly soluble salicylic acid (SA) reduced the non-freezing water content to $0.19-0.17 \mathrm{~g} \mathrm{~g}^{-1}$. On the other hand, matrices composed of highly soluble sodium salicylate (NaSA) showed variable decrease of NFW content- $0.44 \mathrm{~g} \mathrm{~g}^{-1}$ for HF and $0.54-0.51 \mathrm{~g} \mathrm{~g}^{-1}$ for LF and MF. It was also found that the maximum temperature $T_{\max }$ of melting was strongly influenced by the solubility of the drug. Highly soluble NaSA shifted $T_{\max }$ toward the lower negative values, while SA toward the higher positive values. Assessing NFW contents in hydrated HPC matrices may contribute to a better understanding of the drug release and dissolution mechanisms of tablet formulations.
\end{abstract}

Keywords Hydroxypropylcellulose $\cdot$ HPC $\cdot$ Hydration $\cdot$ DSC $\cdot$ Non-freezing water

\section{Introduction}

Hydroxypropylcellulose (HPC) is a non-ionic, water-soluble cellulose ether derivative. It is a semi-crystalline polymer with relatively low $T_{\mathrm{g}}$ and a high degree of amorphous content, which causes high molecular mobility and plasticity [1]. Similarly to other structural analogs of cellulose, HPC can be treated as a natural material, that is biocompatible with human tissues (including blood), non-

Przemysław Talik

mftalik@cyf-kr.edu.pl

1 Department of Inorganic and Analytical Chemistry, Faculty of Pharmacy, Jagiellonian University Medical College, 9 Medyczna St., 30-688 Krakow, Poland toxic and relatively low cost [2]. Its structure with a molar substitution of 3.0 is shown in Fig. 1.

The physical and chemical properties of HPC can be modified by controlling side chain length and branching during the synthesis process. This is possible, because the secondary hydroxyl groups, shown in side chains, are available for further etherification with propylene oxides [3]. This results in formation of structures, containing more than one propylene substituent. The flexibility of HPC synthesis allows it to be obtained in several types characterized by various molecular mass (from 80,000-Klucel ${ }^{\circledR}$ EF Pharm to $1,150,000$ - Klucel ${ }^{\circledR}$ HF Pharm) and viscosity (ranging from 25-Klucel ${ }^{\circledR}$ LF Pharm to 6500-Klucel ${ }^{\circledR}$ MF Pharm mPas). For that reason, HPC, among the other polysaccharides, is widely applied as a drug delivery carrier for oral drug delivery systems in pharmacy [4-8]. It is 


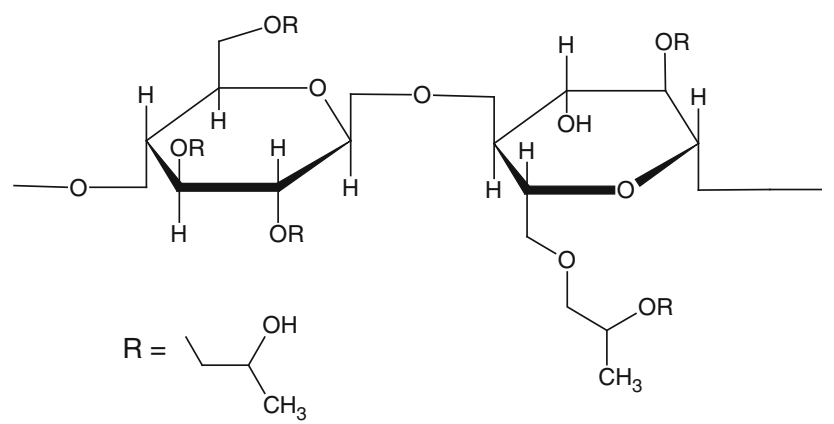

Fig. 1 The structure of hydroxypropylcellulose with a molar substitution of 3.0

also frequently used as a thickener, stabilizer, emulsifier or fat replacer in food industry $[9,10]$. HPC has also found applications in material science and engineering [11-13].

Similarly as in the case of other hydrophilic cellulosebased polymers (cellulose, chitosan, schizophyllan, hyaluronan, CMC) [14], HPC-water interactions are believed to be related to hydrogen bonding, which can lead to formation of new stable structures [15]. These entities are particularly interesting due to their ability to drastically change their mechanical and physicochemical properties. Modern explanation of that peculiar capacity assumes the existence of three distinct fractions of water: free (or bulk) water $(\mathrm{FW})$, freezable bound water $(\mathrm{FBW})$ and non-freezable bound water (NFW) [16]. The first type of water-free water-does not significantly differ in its melting/crystallization temperature and enthalpy from normal (bulk) water. The second type, called freezable bound water, represents the water which is less closely associated with polymer chains. It exhibits a crystallization phase transition and a melting point lower than $0{ }^{\circ} \mathrm{C}$ which distinguishes it from the bulk water. This fraction is also characterized by supercooling and significantly smaller enthalpies than the free water. When first-order phase transitions of small fractions of water (strongly associated with the polymer matrix) cannot be observed calorimetrically, we speak about non-freezable bound water which is the third type. This type of water embedded in hydrophilic polymer does not crystallize even when cooled down to $-100{ }^{\circ} \mathrm{C}$ [17]. For low water contents, all water found in the matrix is considered to be non-freezable [17]. The sum of the freezing bound and non-freezing bound water fractions is the bound water content.

Coexistence of those types of water in the polymer matrix forms a complex, consisting of polymer chains, ice, unfrozen water and air. Both ice and unfrozen water can be embedded in the pores and/or cavities of the matrix. When the dimensions of such a cavity do not exceed several Angstroms, the crystallization of water is difficult or impossible. Liu et al. [18] called such hollow spaces "nanocavities" and demonstrated that they could be an important reason for the formation of non-freezing water. This author concluded that hydrogen bonding is not the sole factor that has influence over water crystallization. Moreover, they suggested that it is more acceptable to think that bound water bind with hydrogen bonds is just one of the various physical states in the polymer.

The existing techniques that can be applied in the research of bound water include, among the others, nuclear magnetic resonance (NMR) [19, 20], magnetic resonance imaging (MRI) [21], time domain reflectometry (TDR) [18] and differential scanning calorimetry (DSC) $[14,15,22-25]$. However, the melting/freezing experiments using DSC seem to be the most suitable to investigate and quantify the NFW.

The method described in $[14,22,24]$ was successfully applied to determine the NFW contents of some raw hydrophilic polysaccharides: cellulose, chitosan and schizophyllan $\left(0.14,0.37\right.$ and $0.38 \mathrm{~g} \mathrm{~g}^{-1}$ respectively), hyaluronan, CMC $90 \mathrm{kDa}$ and CMC700 kDa (0.77, 0.77, $0.84 \mathrm{~g} \mathrm{~g}^{-1}$ respectively) [14].

As described above water holding properties are virtually dependent on the chemical and physical structure of polymer matrix. The objective of this paper was to investigate the non-freezable bound water contents of raw hydroxypropylcellulose Klucel ${ }^{\circledR}$ Pharm HF, MF, and LF by differential scanning calorimetry, three polymers significantly different in their molecular mass and viscosity. Physical mixtures of HPC and low soluble salicylic acid or highly soluble sodium salicylate were used to examine the effect of counter-ions presence and drug solubility on NFW contents.

Assessing NFW contents in hydrated HPC matrices may contribute to a better understanding of the drug release and dissolution mechanisms of tablet formulations.

\section{Experimental}

\section{Materials}

The pharmaceutical Klucel ${ }^{\circledR}$ Pharm hydroxypropylcelluloses of viscosity LF, MF and HF were purchased from Hercules Incorporated, Aqualon Division. Their Brookfield viscosity $\left(\mathrm{H}_{2} \mathrm{O}\right.$, cps), moles of substitution, molecular mass and percent of hydroxypropoxy groups, as quoted by the manufacturer, are collected in Table 1. The salicylic acid (SA) was purchased from Pharma Cosmetic, Krakow, Poland (batch 113383) and sodium salicylate (NaSA) was from Amara, Krakow, Poland (batch 281113) both received as a gift. The water solubility of salicylic acid is $2.2 \mathrm{~g} \mathrm{~L}^{-1}$, while sodium salicylate is nearly 500 times higher. 
Table 1 Basic physicochemical properties of Klucel ${ }^{\circledR}$ Pharm HPC of different viscosity LF, MF and HF

\begin{tabular}{|c|c|c|c|c|c|}
\hline Klucel ${ }^{\circledR}$ HPC & Molecular mass & Moles of substitution & Hydroxypropoxy groups/\% & Viscosity/mPas & At solution concentration $/ \%$ \\
\hline $\mathrm{LF}$ & 95,000 & 3.6 & 72.8 & 126 & 5 \\
\hline MF & 850,000 & 3.8 & 74.8 & 6300 & 2 \\
\hline $\mathrm{HF}$ & $1,150,000$ & 3.8 & 74.5 & 2850 & 1 \\
\hline
\end{tabular}

\section{Sample preparation}

The HPC material was gently mixed for 5 min with the examined drugs using an agate mortar and pestle at a 1:1 polymer/drug w/w ratio to prepare $3 \mathrm{~g}$ of each of the corresponding physical mixtures (PMs). Obtained PMs as well as raw HPC samples were dried in a vacuum desiccator over phosphorus pentoxide for about 4 weeks. Then, portions of about $20-30 \mathrm{mg}$ were accurately weighed and placed into plastic pans. An excess of Milli-Q water was added. The water was allowed to evaporate slowly at room temperature until the desired water content $W_{\mathrm{c}}$ from 0.20 to $5.00 \mathrm{~g} \mathrm{~g}^{-1}$ was obtained. The $W_{\mathrm{c}}$ was defined as water fraction $m_{\mathrm{H} 2 \mathrm{O}}$ related to dry mass $\mathrm{m}_{\text {dry }}$ of HPC or HPC MPs $\left(W_{\mathrm{c}}=m_{\mathrm{H} 2 \mathrm{O}} \times m_{\mathrm{dry}}^{-1}\right)$. The aluminum sample pans were exposed to steam in an autoclave at $120{ }^{\circ} \mathrm{C}$ for $3 \mathrm{~h}$, to form a passive coat of $\mathrm{Al}_{2} \mathrm{O}_{3}$, for protection from spontaneous reactions with water. Then, the homogeneous material of about $8 \mathrm{mg}$ was transferred to pans, hermetically sealed and conditioned at room temperature for about $30 \mathrm{~h}$ to obtain an equilibrium state. The crucibles were weighted before and after each measurement to insure that there is no loss of mass.

\section{Thermal method}

The qualitative and quantitative studies of hydrated polymers using DSC are based on freezing and thawing experiments, in which the difference in physical properties between FBW in form of ice and NFW is investigated. As previously suggested in [22] and also successfully applied in $[14,24]$, the measured enthalpies of melting were first normalized to the mass of the dry raw HPC or their PMs and then plotted against the respective water concentration $\left(W_{\mathrm{c}}\right)$. In this way, the linear dependences $\Delta H=\mathrm{f}\left(W_{\mathrm{c}}\right)$ were obtained and the NFW contents were estimated by extrapolation to $\Delta H=0$.

\section{DSC measurements}

DSC analysis was performed with DSC 7020 calorimeter, Hitachi Inc., equipped with DSC 7020 electric cooling unit. The apparatus was calibrated with indium, tin standards (purity of 99.9999\%) and Milli-Q water. The freezing and heating measurements were performed in nitrogen atmosphere with a flow rate of $50 \mathrm{~mL} \mathrm{~min}{ }^{-1}$ in three steps: cooling from $20{ }^{\circ} \mathrm{C}$ to $-55{ }^{\circ} \mathrm{C}$ at $3{ }^{\circ} \mathrm{C} \mathrm{min}{ }^{-1}$, isothermal at $-55{ }^{\circ} \mathrm{C}$ for $2 \mathrm{~min}$ and heating back to $20^{\circ} \mathrm{C}$ at $3{ }^{\circ} \mathrm{C} \mathrm{min}^{-1}$. In order to reduce experimental errors, DSC runs were repeated three times.

The obtained plots were interpreted using Muse Measurement $\mathrm{v}$ 9.21U software. The determination of enthalpies was carried out using a T-Slice Analysis (Integral Tangential) taking into account the baseline shift and nonlinearity. The melting temperatures of water absorbed in the sample were determined at the maximum point of the corresponding enthalpy peaks $\left(T_{\max }\right)$.

\section{Results and discussion}

To achieve improved readability upper parts of curves corresponding to crystallization were cut off, because nearly all of them showed a large "loop". This artifact is shown in Fig. 2 and is attributed to the exothermic processes during water crystallization.

Figure 3 shows some representative examples of DSC runs, obtained from hydrated, raw HPC HF samples of $W_{\mathrm{c}}$ ranging from 0.56 to $2.46 \mathrm{~g} \mathrm{~g}^{-1}$. The first curve marked as $W_{\mathrm{c}}=0.56$ can be considered a straight baseline because no significant phase transition of water is observed. It can be explained by the fact that for low concentrations of water, nearly all absorbed molecules of non-freezable water

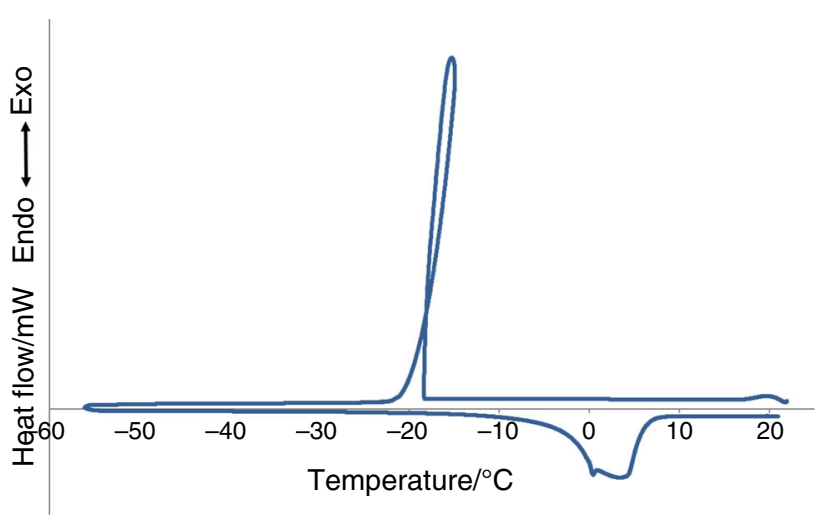

Fig. 2 The DSC curve of HPC MF sample with an artifact in the form of a large "loop" in the central part of trimmed curve 


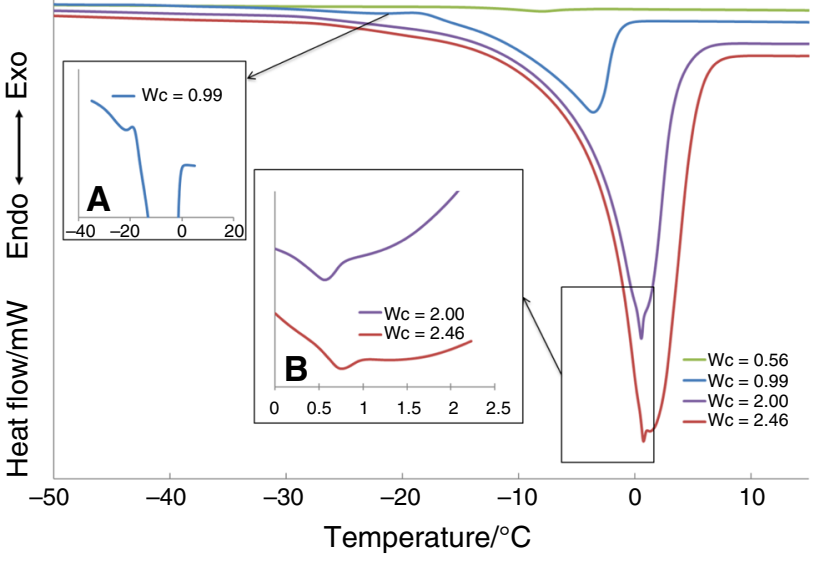

Fig. 3 The DSC melting curves, obtained from raw HPC HF/water samples of $W_{\mathrm{c}}$ ranging from 0.56 to 2.46. The arrows point a magnified small and broad peak obtained for sample with $W_{\mathrm{c}}=0.99$ (subfigure a) and two overlapped peaks obtained for samples with $W_{\mathrm{c}}=2.00$ and 2.46 (subfigure $\mathrm{b}$ )

(0.56 $\mathrm{g}$ of water per $1.00 \mathrm{~g}$ of HPC HF) diffuse into the amorphous region of the sample and each of them is being restrained by the hydroxyl group of hydroxypropylcellulose chain. When the quantity of water in the matrix is slightly increased, the first-order transition of crystallization is absent, while the melting peak can be seen. This happens unless the water content exceeds a certain $W_{\mathrm{c}}$ amount, which varies in accordance with the chemical structure of polysaccharide and/or additives. When this value is reached, typically two separate melting peaks are observed, usually with a very distant $T_{\max }$. That phenomenon is highlighted in Fig. 3 (subfigure a). The first peak on $W_{\mathrm{c}}=0.99$ at the temperature $T_{\max }=-21.6^{\circ} \mathrm{C}$ was very small and broad and the second, well formed at $T_{\max }=-3.6^{\circ} \mathrm{C}$. With higher contents of water, the peaks were shifted to a higher temperature range. In most of cases, only one broad endothermic event (two overlapped peaks) could be seen $[17,24]$. However, rarely the peaks were separated and that is shown on subfigure $b$. The water molecules originating from ice which melts at higher temperatures (around $0{ }^{\circ} \mathrm{C}$ ) are assigned to a free water, and those that melt at proportionally lower temperatures are assigned to the freezable bound water [17].

In Fig. 4, a relationship between maximum temperatures $T_{\text {max }}$ of melting of water vs. appropriate $W_{\mathrm{c}}$, obtained from HPC and their mixtures is shown.

Figure 4A gathers LF, MF and HF types of raw hydroxypropylcellulose. One can see that all lines are very close to each other and form a cluster. This means that the internal architecture of the samples was comparable and nearly the same number of hydroxyl groups interacts with similar water molecules. The effect of polymer mass and viscosity is negligible. Further analysis of Fig. 4a shows that the cluster passes the $0{ }^{\circ} \mathrm{C}$ axis (red dashed line)
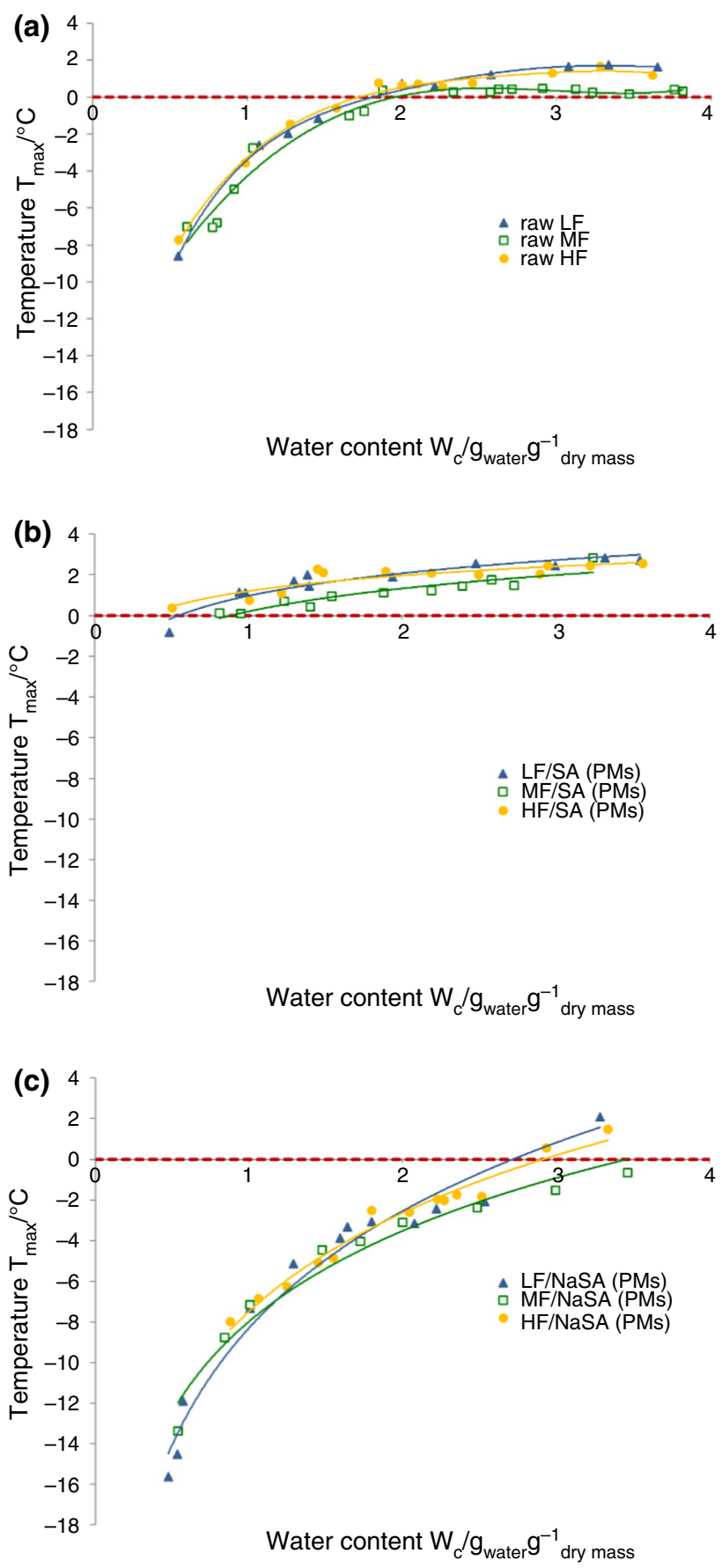

Fig. 4 Relationship between maximum temperatures $T_{\max }$ of melting of water and its content $W_{\mathrm{c}}$, obtained from HPC LF (blue triangle), MF (blank green square), HF (yellow circle) and their mixtures with examined drugs (PMs). a-raw HPC LF, MF, HF; b-HPC LF, MF, HF mixtures with low solubility drug: salicylic acid (SA); c-HPC LF, MF, HF mixtures with high solubility drug: sodium salicylate (NaSA). Horizontal red dashed line indicates $0^{\circ} \mathrm{C}$. For comparison purposes, the $W_{\mathrm{c}}$ and $T_{\max }$ axes were fixed for all graphs. (Color figure online)

between $W_{\mathrm{c}}$ around 1.75 and 1.85 , after a rapid increase from about $-9{ }^{\circ} \mathrm{C}$. Then, the lines reach a plateau and do not pass $2{ }^{\circ} \mathrm{C}$ until the end of the measurement range. The 
sole exception is HPC MF which goes along $0{ }^{\circ} \mathrm{C}$ axis. Figure $4 \mathrm{~b}$ collects all used types of HPC and low solubility salicylic acid physical mixtures. As in the case of raw material, the HPC MF/SA PM is slightly under the rest of lines; however, the distance does not exceed $1.1^{\circ} \mathrm{C}$. In the whole range of $W_{\mathrm{c}}$, the $T_{\max }$ were positive and the cluster gently rose from $0{ }^{\circ} \mathrm{C}$ not exceeding $3{ }^{\circ} \mathrm{C}$ $\left(\Delta T_{\max } \approx 3{ }^{\circ} \mathrm{C}\right)$. This corresponds very well with the observations of Faroongsarng and Sukonrat [27], who showed that the depression of melting temperature is not only attributed to water-polymer interactions, but water embedded in the pores and/or cavities of the matrix as well. In HPC/SA mixtures, both phenomena take place. There are less hydroxyl groups when compared to raw HPCs and access to them is more restricted in the presence of SA molecules. The opposite effect can be seen in Fig. 4c, where HPC mixtures with high soluble sodium salicylate (NaSA) were studied. The lines are not as bundled as raw HPC or SA PMs samples, and they are crossing each other. The maximum temperatures of the group begin between -16 and $-12{ }^{\circ} \mathrm{C}$ and are negative in the whole measurement range $\left(\Delta T_{\max } \approx 12-16^{\circ} \mathrm{C}\right)$. Considering that the architecture of a matrix $(1: 1 \mathrm{w} / \mathrm{w} \mathrm{HPC} / \mathrm{NaSA})$ is similar or even identical to that of salicylic acid, such interactions must be related to a strong influence of an easily dissociated sodium cation.

The determination of non-freezable bound water content is possible using both the crystallization or melting enthalpies. However, obtained values are not equal and the NFW contents determined using crystallization enthalpies are smaller than those calculated from melting enthalpies. That phenomenon was formerly explained and discussed by Mlčoch and Kučerík [14]. In their publication, the authors demonstrated that the difference in enthalpy of crystallization and melting is related to the properties of water and is "hidden" in the segment of the curve where the heating is switched into cooling part.

To complete these conclusions, we want to point at mentioned above artifact (the "loop") that appeared during the cooling. Certainly, this is another important factor which affects the final measurements. To avoid this problem, we tried to lower the cooling rate or decrease the sample mass. However, it did not bring the expected result. For that reason, only the cooling parts of the DSC curves were used in further work.

The measured melting enthalpies of hydrated raw HPC samples and their PMs were plotted against respective $W_{\mathrm{c}}$ and a very good fit with the linear $\Delta H=\mathrm{f}\left(W_{\mathrm{c}}\right)$ regression (as it is shown in Fig. 5) was obtained. The linear trend was maintained throughout the measuring range.

The calculated values of NFW and accompanying coefficients of determination $\mathrm{R}^{2}$ are listed in Table 2.

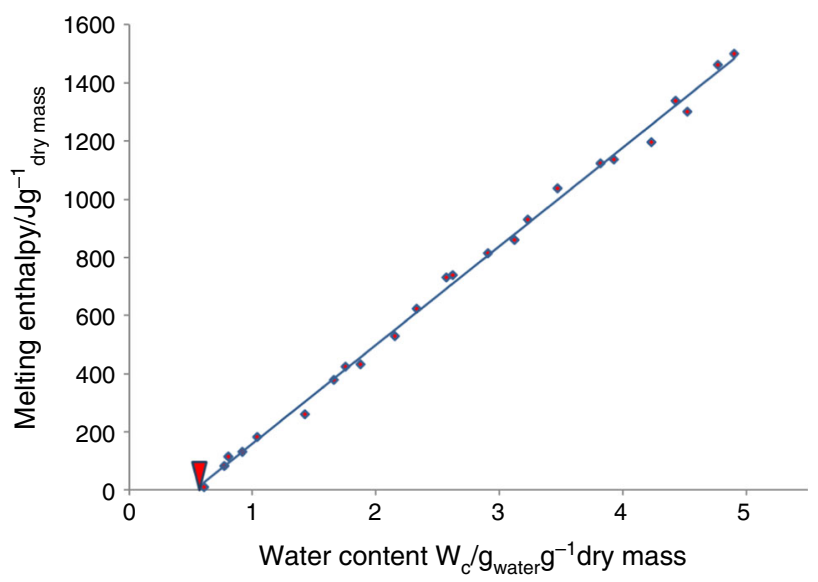

Fig. 5 The determined enthalpies of melting versus respective contents of water $W_{\mathrm{c}}$ represented linear dependences. The sought value of non-freezing water calculated as the concentration at which $\Delta H=0$ (extrapolation) is marked with red triangle. The figure shows an example obtained for $\operatorname{HPC} \operatorname{MF}\left(\Delta H=-182.5+337.7 \times W_{\mathrm{c}}\right)$

Table 2 Contents of non-freezing water determined for raw HPC samples and their physical mixtures with examined drugs of different solubility: salicylic acid (SA) — low solubility drug, sodium salicylate (NaSA) - high solubility drug; $n_{\mathrm{NFW}}$ stands for the number of water molecules per disaccharide HPC unit

\begin{tabular}{lllr}
\hline HPC sample & $R^{2}$ & NFW/gg & $n_{\mathrm{NFW}}$ \\
\hline LF & 0.9928 & 0.54 & 22.2 \\
MF & 0.9922 & 0.54 & 22.9 \\
HF & 0.9936 & 0.51 & 21.7 \\
LF/SA & 0.9992 & 0.19 & 7.8 \\
MF/SA & 0.9949 & 0.18 & 7.7 \\
HF/SA & 0.9927 & 0.17 & 7.2 \\
LF/NaSA & 0.9970 & 0.50 & 20.6 \\
MF/NaSA & 0.9972 & 0.48 & 20.4 \\
HF/NaSA & 0.9966 & 0.44 & 18.7 \\
\hline
\end{tabular}

As seen in Table 2, the contents of non-freezing water in different LF, MF, HF types of raw HPC are nearly equal and range from 0.51 to $0.54 \mathrm{~g} \mathrm{~g}^{-1}$. This indicates that the molecular mass and viscosity do not affect the content of non-freezing water. In this case, the access to hydroxyl groups and their amount, which is the same, plays the key role (see Table 1). For the salicylic acid physical mixtures, the non-freezing water contents were also similar, but ranging from 0.17 to $0.19 \mathrm{~g} \mathrm{~g}^{-1}$ (3 times smaller comparing to their raw analogs). This confirms earlier research [18], where authors demonstrated, that apart from hydrogen bonding, also the presence of "nanocavities" influences the formation of non-freezing water. In other words, such a threefold decrease may be explained by the fact that with the lowering amount of hydroxyl groups, the number of 
"nanocavities" also decreases. That is because the "nanocavities" were occupied or there was limited access to them due to small salicylic acid molecules.

The last group of samples under study was HPC and NaSA matrices. What can be considered interesting is that the NFW contents were similar to unmixed HPC. The LF and MF types were nearly similar and equaled to 0.50 and $0.48 \mathrm{~g} \mathrm{~g}^{-1}$, respectively, HF was $0.44 \mathrm{~g} \mathrm{~g}^{-1}$. This clearly shows that the deficiency of hydroxyl groups was compensated by the strong influence of the counter ion $\mathrm{Na}^{+}$ [26]. High dissociation leads to a stronger interaction between the dissociated ion pair and water molecules. This phenomenon activates adsorption of bound water.

The number of water molecules per disaccharide unit $\left(n_{\mathrm{NFW}}\right)$ calculated for all types of raw HPC was in the range of 21.7-22.9 and was comparable to $n_{\mathrm{NFW}}$ obtained for CMC $700 \mathrm{kDa} 21.9$ [14]. The other studied polymers such as cellulose, chitosan, schizophyllan, hyaluronan and CMC $90 \mathrm{kDa}$ have $n_{\mathrm{NFW}}$ lower than 18.7 (2.4, 7.00, 14.1, 17.2, 18.7 , respectively).

\section{Conclusions}

In the present study, differential scanning calorimetry was used to investigate the non-freezing water contents of hydroxypropylcellulose of various molecular mass and viscosities: HF, MF, LF and their matrices composed of drugs with different solubilities such as salicylic acid and sodium salicylate. It was found that under the measurement conditions, the viscosity and molecular mass affects the NFW contents in a raw polymer only negligibly. Poorly soluble small molecule drug (salicylic acid) reduced the NFW amount nearly 3 times. It was demonstrated that the formation of non-freezable water is influenced by both the reduced access to a smaller number of hydroxyl groups and the spatial architecture ("nanocavity") of the polymer. The NFW contents calculated for matrices with highly soluble sodium salicylate were comparable to those of raw HPC, which shows how strong an influence of the counter $\mathrm{Na}^{+}$ ion is. During the studies, it was also found that the maximum temperature $T_{\max }$ of melting was strongly influenced by the solubility of the drug. Highly soluble NaSA shifted $T_{\max }$ toward the lower negative values, while SA toward the higher positive values.

This type of research may contribute to better understanding of processes that influence dissolution behavior of both highly and poorly soluble drugs from tablet formulations.

Open Access This article is distributed under the terms of the Creative Commons Attribution 4.0 International License (http://creative commons.org/licenses/by/4.0/), which permits unrestricted use, distribution, and reproduction in any medium, provided you give appropriate credit to the original author(s) and the source, provide a link to the Creative Commons license, and indicate if changes were made.

\section{References}

1. Picker-Freyer KM, Durig T. Physical mechanical and tablet formation properties of hydroxypropylcellulose: in pure form and in mixtures. AAPS PharmSciTech. 2007;8(4):E1-9.

2. Alderman DA. A review of cellulose ethers in hydrophilic matrices for oral controlled-release dosage forms. Int J Pharm Technol Prod Manuf. 1984;5:1-9.

3. Klose RE, Glickman M. Gums. In: Furia TE, editor. Handbook of food additives. 2nd ed., Tom 1. CRC Press: Boca Raton London, New York: Washington D.C.; 1973. p. 323.

4. Tanaka A, Furubayashi T, Tomisaki M, Kawakami M, Kimura S, Inoue D, Kusamori K, Katsumi H, Sakane T, Yamamoto A. Nasal drug absorption from powder formulations: the effect of three types of hydroxypropyl cellulose (HPC). Eur J Pharm Sci. 2017; 96:284-9.

5. Macchi E, Zema L, Pandey P, Gazzaniga A, Felton LA. Influence of temperature and relative humidity conditions on the pan coating of hydroxypropyl cellulose molded capsules. Eur J Pharm Biopharm. 2016;100:47-57.

6. Hughey JR, Keen JM, Bennett RC, Obara S, McGinity JW. The incorporation of low-substituted hydroxypropyl cellulose into solid dispersion systems. Drug Dev Ind Pharm. 2015;41(8): 1294-301.

7. Bajdik J, Regdon G Jr, Marek T, Eros I, Süvegh K, Pintye-Hódi $\mathrm{K}$. The effect of the solvent on the film-forming parameters of hydroxypropyl-cellulose. Int J Pharm. 2005;301(1-2):192-8.

8. Ye R, Harte F. High pressure homogenization to improve the stability of casein-hydroxypropyl cellulose aqueous systems. Food Hydrocoll. 2014;35:670-7.

9. Ye R, Cheng Q, Cai J, Feng T, Wang G. Stable casein-hydroxypropyl cellulose complexes at low pH. J Food Qual. 2016;39(4): 292-300.

10. Liu X, Zhou Y, Nie W, Song L, Chen P. Fabrication of hydrogel of hydroxypropyl cellulose (HPC) composited with graphene oxide and its application for methylene blue removal. J Mater Sci. 2015;50(18):6113-23.

11. Ledwon P, Andrade JR, Lapkowski M, Pawlicka A. Hydroxypropyl cellulose-based gel electrolyte for electrochromic devices. Electrochim Acta. 2015;159:227-33.

12. Barzic AI, Dimitriu DG, Dorohoi DO. New method for determining the optical rotatory dispersion of hydroxypropyl cellulose polymer solutions in water. Polym Eng Sci. 2015;55(5):1077-81.

13. Vueba ML, Batista de Carvalho LA, Veiga F, Sousa JJ, Pina ME. Influence of cellulose ether mixtures on ibuprofen release: MC25, HPC and HPMC K100 M. Pharm Dev Technol. 2006;11(2): 213-28.

14. Mlčoch T, Kučerík J. Hydration and drying of various polysaccharides studied using DSC. J Therm Anal Calorim. 2013;113(3): 1177-85.

15. Hatakeyama T, Inui $Y$, Iijima $M$, Hatakeyama $H$. Bound water restrained by nanocellulose fibres. J Therm Anal Calorim. 2013;113(3):1019-25.

16. Wolfe J, Bryant G, Koster KL. What is'unfreezable water', how unfreezable is it and how much is there? CryoLetters. 2002;23: 157-66.

17. Ping ZH, Nguyen QT, Chen SM, Zhou JQ, Ding YD. States of water in different hydrophilic polymers-DSC and FTIR studies. Polymer. 2001;42:8461-7. 
18. Liu GL, Yao KD. What causes the unfrozen water in polymers: hydrogen bonds between water and polymer chains? Polymer. 2001;42:3943-7.

19. Watanabe K, Wake T. Measurement of unfrozen water content and relative permittivity of frozen unsaturated soil using NMR and TDR. Cold Reg Sci Technol. 2009;59:34-41.

20. Průšovă A, Conte P, Kučerík J, Alonzo G. Dynamics of hyaluronan aqueous solutions as assessed by fast field cycling NMR relaxometry. Anal Bioanal Chem. 2010;397:3023-8.

21. Kulinowski P, Młynarczyk A, Jasiński K, Talik P, Gruwel ML, Tomanek B, Węglarz WP, Dorożyński P. Magnetic resonance microscopy for assessment of morphological changes in hydrating hydroxypropylmethylcellulose matrix tablets in situ-is it possible to detect phenomena related to drug dissolution within the hydrated matrices? Pharm Res. 2014;31(9):2383-92.

22. Liu J, Cowman MK. Thermal analysis of semi-dilute hyaluronan solutions. J Therm Anal Calorim. 2000;59:547-57.
23. Dehabadi L, Udoetok IA, Wilson LD. Macromolecular hydration phenomena: an overview of DSC studies on sulfonated tetrafluoroethylene-based fluoropolymer-copolymer (Nafion) and cellulose biopolymer materials. J Therm Anal Calorim. 2016;126(3):1851-66.

24. Průšovă A, Šmejkalovă D, Chytil M, Velebný V, Kučerík J. An alternative DSC approach to study hydration of hyaluronan. Carbohydr Polym. 2010;82:498-503.

25. Hatakeyama T, Tanaka M, Hatakeyama $H$. Thermal properties of freezing bound water restrained by polysaccharides. J Biomater Sci Polym Ed. 2010;21:1865-75.

26. Berthold J, Desbrières J, Rinaudo M, Salmén L. Types of adsorbed water in relation to the ionic groups and their counter-ions for some cellulose derivatives. Polymer. 1994;35(26):5729-36.

27. Faroongsarng D, Sukonrat P. Thermal behavior of water in the selected starch- and cellulose-based polymeric hydrogels. Int $\mathbf{J}$ Pharm. 2008;352(1-2):152-8. 